\title{
Study of the Electronic Current Transformer's Additional Error Testing
}

\section{Chun-Yan YANG ${ }^{1, a}$, Deng-Yun LI ${ }^{1, b,}$, , Hao-Liang HU ${ }^{1 . c}$, Hui PENG ${ }^{2, d}$, Li $Y A O^{3, e}$, Tao XU ${ }^{3, f}$}

${ }^{1}$ China Electric Power Research Institute, Wuhan, China;

2Wuhan University, Wuhan, China;

${ }^{3}$ State Grid Zhejiang Electric Power Company Electric Power Research Institute, Hangzhou, China ayangchunyan@epri.sgcc.com.cn,b2411756356@qq.com,chuhaoliang@epri.sgcc.com.cn

dpenghui@whu.edu.cn,eyaoli@epri.sgcc.com.cn,,xutao@epri.sgcc.com.cn

\section{${ }^{*}$ Corresponding author}

Keywords: electronic voltage transformer, error test, temperature characteristics, primary connection characteristics, harmonic characteristics

Abstract: The measurement accuracy of the electronic current transformer are susceptible to environmental factors and interference of external magnetic field. Based on analyzing the effect of temperature, primary wiring and harmonic factor on the measurement accuracy of current transformer of rogowski coil and full optical fiber current transformer, this paper formulated corresponding protocol and tested the additional error of two kinds of electronic current transformers. After comparison and analysis of experimental data, this paper got the error characteristics of temperature, primary wiring and harmonics.

\section{Introduction}

Compared to traditional current transformers, electronic current transformers eliminate the phenomenon of magnetic saturation in the measurement process [1-3]. Although the electronic current transformer has many advantages, its performance is easily affected by the external environmental factors[4-6].This paper analyzed the influence of environmental factors on measurement error of electronic current transformer firstly. Then formulated the corresponding experimental program and test the additional error of rogowski-coil current transformer and all fiber optical current transformer.

\section{Principle and Error Analysis of Electronic Current Transformer}

\section{Principle and Error Analysis of Optical Current Transformer}

Optical current transformer is mainly based on the principle of Faraday magneto-optical effect. As shown in Fig. 1[7,8], when a beam of polarized light incident to the magneto-optical medium, the polarization plane deflected. The rotation angle is proportional to the magnetic field intensity.

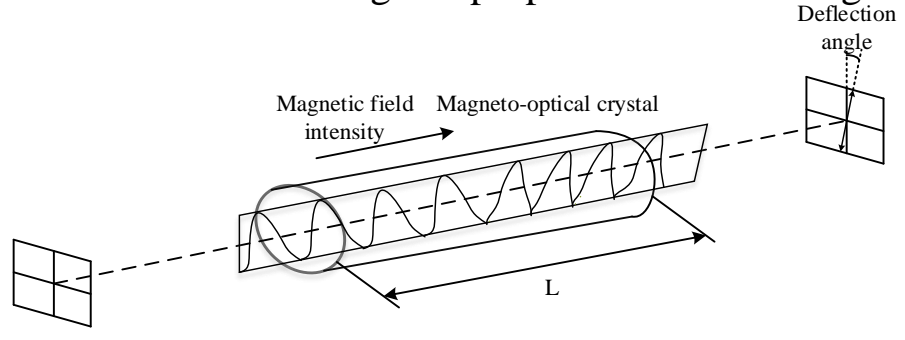

Fig. 1 Faraday magneto-optical effect diagram

The error of optical current transformer is influenced by many factors in the process of operation. This paper analyzed the influence of temperature change, primary wiring and harmonic on the error of all fiber current transformer.

(1) The effect of temperature on measurement error 
The change in temperature results in changes in the light source operating wavelength and the refractive index of the optical material, then cases the change of the Verdet constant. In addition, the change of temperature affect operation characteristics of polarization maintaining fiber 1/4 wave plate, sensing fiber loop and other optical devices, which will change the measurement accuracy.

(2) The effect of primary wiring on measurement error

All fiber optic current transformer uses the multi turn optical fiber loop to measure the current. For any interfering magnetic field generated by adjacent conductors, the integral of any closed loop vector is zero. Theoretically, adjacent conductors and ambient electromagnetic fields can scarcely effect the measurement error of the fiber optical current transformer.

(3) The effect of harmonic on measurement error

When the frequency of the power grid is shifted, the phase shift parts and the filtering parts in the signal processing circuit of the optical current transformer will be affected. The amplitude and phase of the output signal will change with the frequency.

\section{Principle and Error Analysis of Active Current Transformer}

Typical rogowski-coil structure is shown in Fig. 2. The skeleton is annular and Skeleton section is a flat rectangular or circular. The coil is uniformly wound on the skeleton and the measured current passes through the center of the loop.

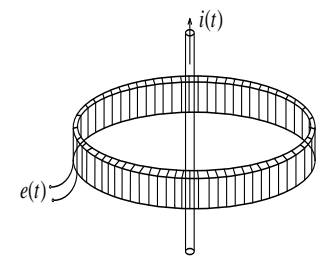

Fig.2 Rogowski coil structure diagram

When the measured current is changed, the induced electromotive force generated by the coil can be calculated by Formula (2) under ideal conditions.

(2)

$$
e(t)=-\mu_{0} n S \cdot \frac{d i}{d t}=-M \cdot \frac{d i(t)}{d t}
$$

$\mathrm{n}$ is Line turn density, $\mu_{0}$ is permeability of vacuum, $\mathrm{S}$ is the cross-sectional area of the coil.

Formula (2) shows that the induced electromotive force of the coil is proportional to the measured current change rate. The quantity which has a linear relationship with the measured current can be obtained by integrating $e(t)$. This paper focuses on the additional measurement error of electronic current transformer based on rogowski coil. Firstly, analyzed the influence of temperature, primary wiring and harmonic wave on current transformer.

(1) The effect of temperature

The parts of phase compensation, analog-to-digital conversion, and electro-optical conversion of electronic current transformer based on rogowski coil need electronic circuits to realize. Electronic circuits are susceptible to the change of temperature. The effect of temperature on electronic circuits is a comprehensive response which must be tested with the whole transformer.

(2) The effect of primary wiring

It's difficult to meet the ideal conditions that the wire to be measured is infinitely long and the line turns are uniform and symmetrical. In addition, the adjacent phase, circuit breaker, and disconnecting link which generate strong electromagnetic will affect the accuracy of current transformer.

(3) The effect of harmonic

When measuring the current, the electronic current transformer based on rogowski coil needs obtain the differential signal of measured current firstly. Then gains the voltage signal proportional to the measured current by integrating. High order harmonic component of the signal to be measured may cause A/D converter saturated and loss measurement information. 


\section{Test Methods for Electronic Current Transformer Error}

The circuit arrangement of electronic current transformer error test is shown in Fig.3. The measured wire gets through the current transformer's center. The measured current is generated by the power frequency current source and current booster. The standard current transformer is connected with the tested electronic current transformer in series. The ratio and the angle difference of the electronic current transformer are acquired by the calibrator.

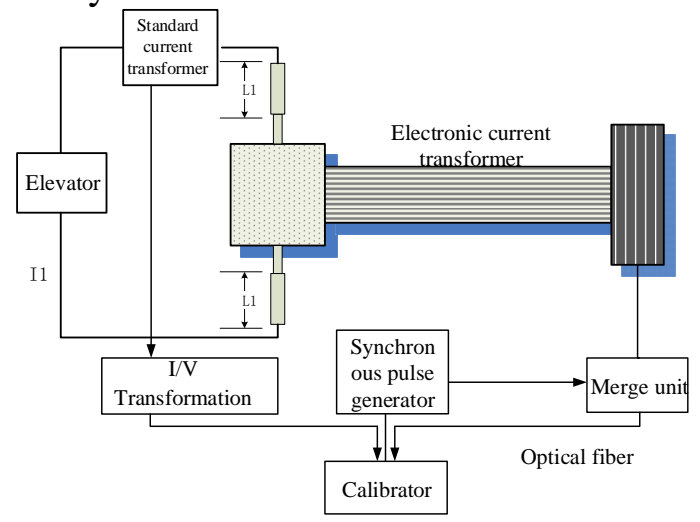

Fig. 3 Wiring diagram of error test

(1) Error test method for temperature characteristics

The circuit arrangement is shown in Fig. 3. Put the electronic current transformer in the temperature control room before the experiment. Under the condition of rated current, the temperature gradient is set to 4 degrees per hour. Then test the ratio error and angle error under the rated current every 15 minutes and record the temperature of environment and temperature control chamber at the mean time. In order to detect the accuracy of electronic current transformer dynamically, two measuring points are added to each ambient temperature during heating and cooling.

(2) Error test method for primary wiring

In the test circuit shown in Fig. 3, the current bus deviate from the center of electronic current transformer. Two electronic current transformers with the same principle and structure are added respectively on both sides of the test sample. Three current transformers placed side by side and kept a certain distance.

(3) Error test method for harmonic

The principle of the test circuit is shown as Fig. 4. The harmonic current generator produces the current superposed by fundamental current and harmonic current. The measured electronic current transformer are connected in series with a coaxial shunt used for tracing. The calibrating device receives the signal of the electronic current transformer and the coaxial diverter. After the spectrum analysis and measurement error analysis, calculate the amplitude error and ratio error under each specified frequency.

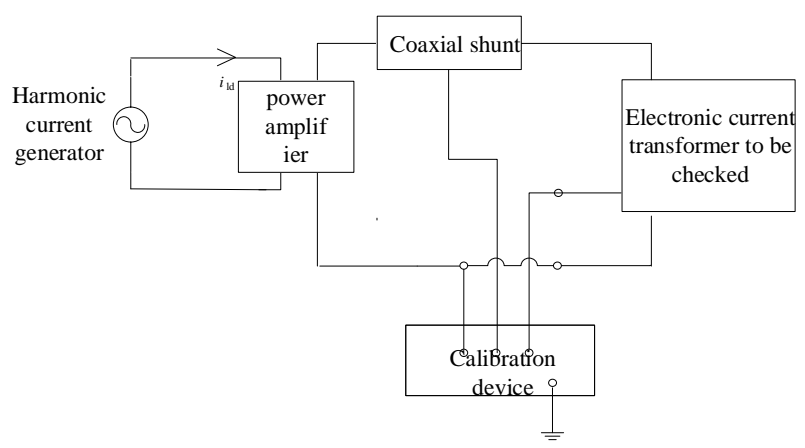

Fig.4 Schematic diagram of harmonic test 


\section{The Test Results of Electronic Current Transformer Error Characteristics}

\section{The Test Results of Optical Current Transformer}

Select three fiber optical current transformers as the sample to complete the temperature characteristic test. The sample's parameters are shown below: the rated current is $50 \mathrm{~A}$, the rated frequency is $50 \mathrm{~Hz}$, the accuracy level is 0.5 . The test results as shown in Fig. 5.
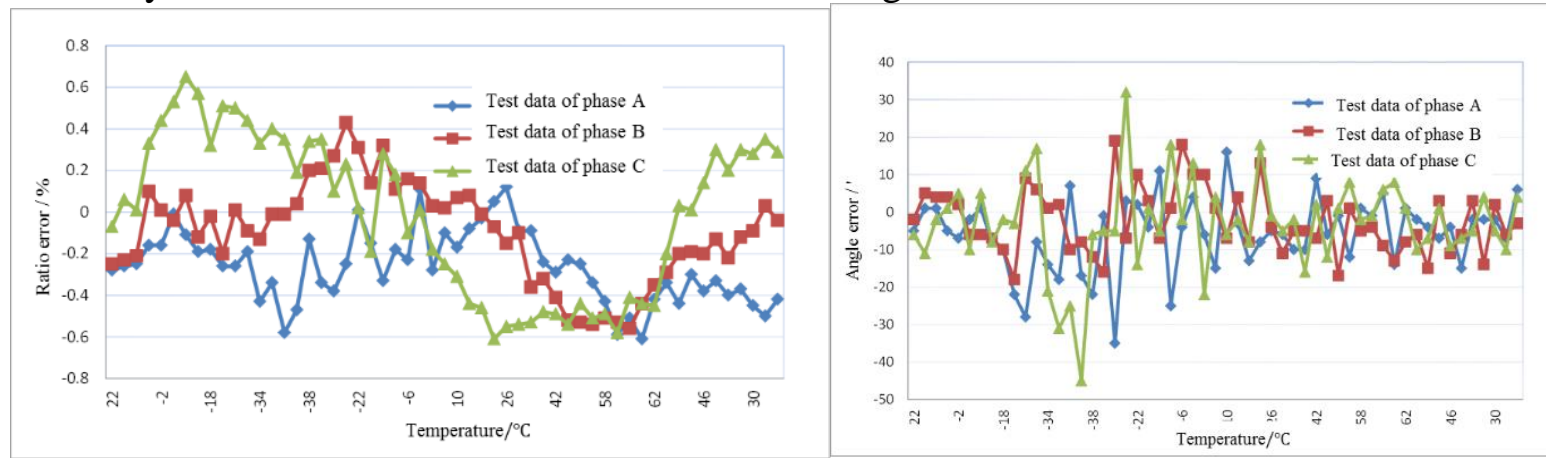

Fig. 5 The ratio error and angle error of FOCT caused by the change of temperature

The results show that the error trend of three fiber optical current transformers are different. And the temperature characteristics of fiber optical current transformer have no obvious regularity.

The test results of primary wiring' effect on measurement error are as follows:
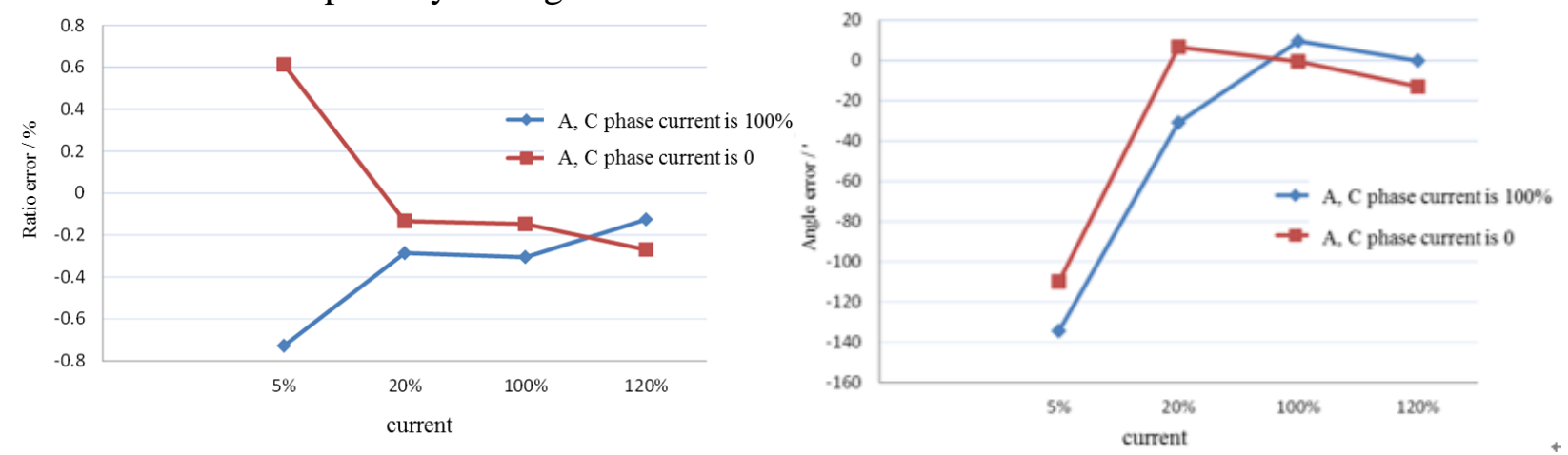

Fig. 6 The ratio error and angle error of FOCTr caused by the style of primary wiring

The ratio and the angle error of FOCT are all out of the error limit. When the current is $5 \%$ of the rated current, the variation of ratio error influenced by the adjacent magnetic field is $1.3 \%$; when the current is greater than $20 \%$ of the rated current, the variation of ratio error is within $0.2 \%$. The ratio error is less affected by the adjacent magnetic field. When the current is $20 \%$ of the rated current, the variation of angle error influenced by the adjacent magnetic field is 40 '; when the current is greater than $20 \%$ of the rated current, the angle error is less affected by the adjacent magnetic field.

In the process of test for the effect of harmonic wave on measurement error, the contents of three times and five times frequency harmonic are respectively about $20 \%$ and $4 \%$ of the fundamental wave. The contents of eleven times and fifteen times frequency harmonic are both $3 \%$ of the fundamental wave. The results of test are as follows: 


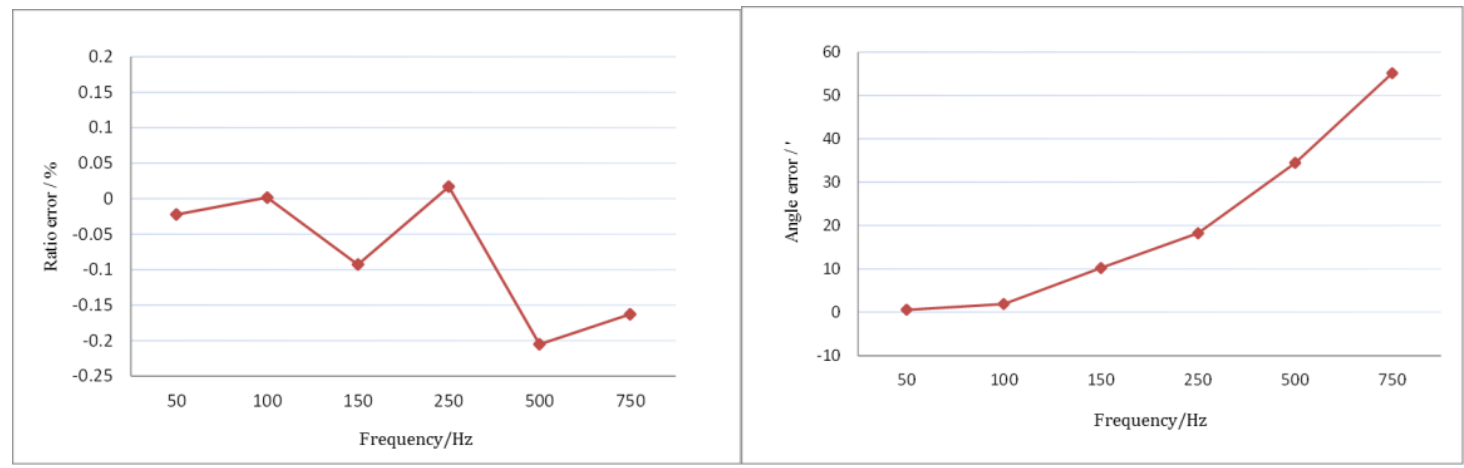

Fig. 7 The ratio error and angle error of FOCT caused by the harmonic

The test results show that the ratio error and angle error become larger with the increase of harmonic frequency. This phenomenon may be related to the optical path and signal processing circuit of optical current transformer.

\section{The Test Results of Active Electronic Current Transformer Error}

Select three electronic transformers based on rogowski coil as the sample to complete the error characteristic test. The sample's parameters are shown below: the rated current is 50A, the rated frequency is $50 \mathrm{~Hz}$, the accuracy level is $0.2 \mathrm{~S}$.

The temperature characteristics test results of measurement error is shown in Fig. 8.
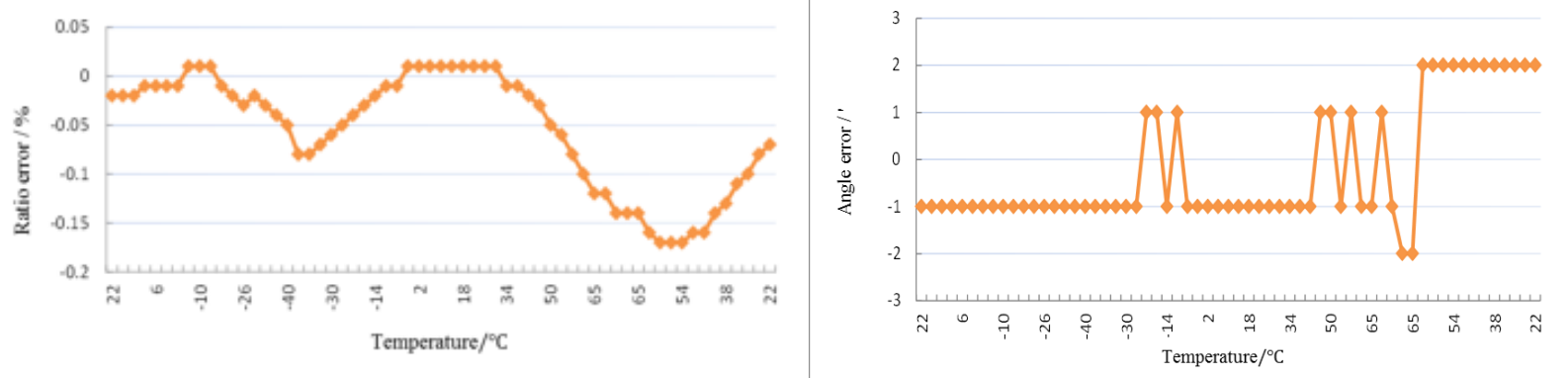

Fig. 8. The ratio error and angle error of active electronic current transformer caused by the change of temperature

The test results show that the error of the capacitive voltage transformer is within the error limit in the full temperature range. The ratio error is basically stable with the change of temperature in the range from $0{ }^{\circ} \mathrm{C}$ to $30^{\circ} \mathrm{C}$, so is the angle error in the range from $2{ }^{\circ} \mathrm{C}$ to $40^{\circ} \mathrm{C}$ and $-10^{\circ} \mathrm{C}$ to $-30^{\circ} \mathrm{C}$. But the angle error is sensitive to the change of temperature in the range from $-30^{\circ} \mathrm{C}$ to $-14^{\circ} \mathrm{C}$ and from $40^{\circ} \mathrm{C}$ to $65^{\circ} \mathrm{C}$.

The measurement error test results of primary wiring is shown in Fig. 9.
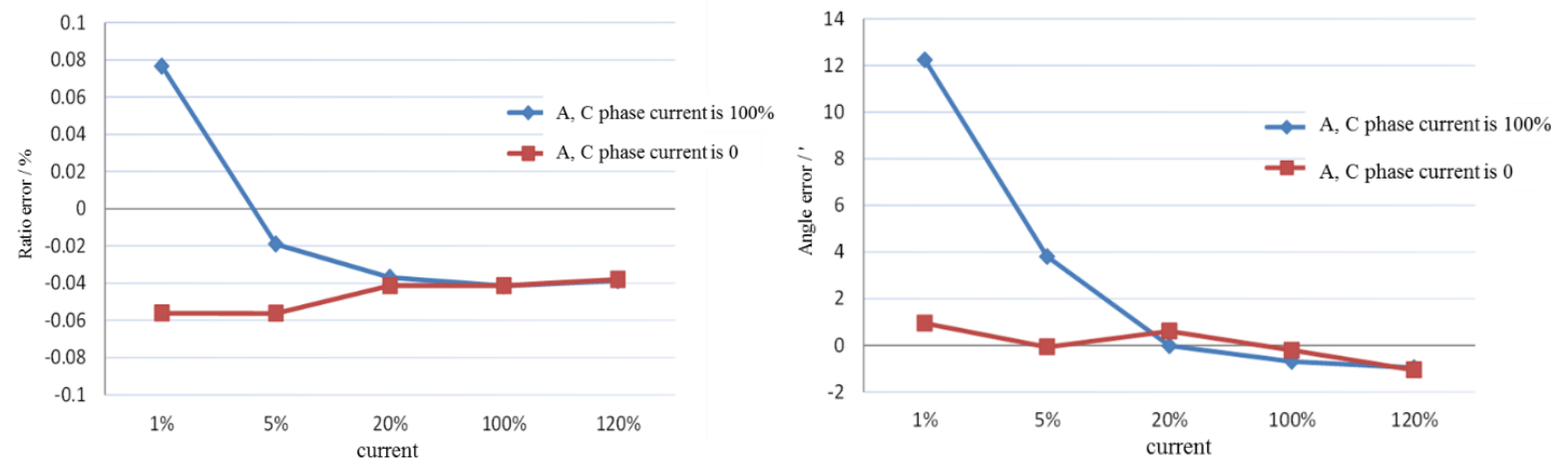

Fig. 9 The ratio error and angle error of active electronic current transformer caused by the style of primary wring 
When the current is $1 \%$ of the rated current, the variation of ratio error is about $0.14 \%$ and the variation of angle error is about 11'; When the current is $5 \%$ of the rated current, the variation of ratio error is about $0.04 \%$ and the variation of angle error is about 4 ; In the condition of $20 \%$ and $120 \%$ of the rated current, the two error curve coincide nearly which shows that the adjacent magnetic field has no effect on the error when the current is greater than $20 \%$ of the rated current.

Then test the effect of the harmonic on error characteristics. The contents of three times, five times and seven times frequency harmonic are respectively about $5 \%, 4 \%$ and $3 \%$ of the fundamental wave content. The test results are shown as Fig. 10.
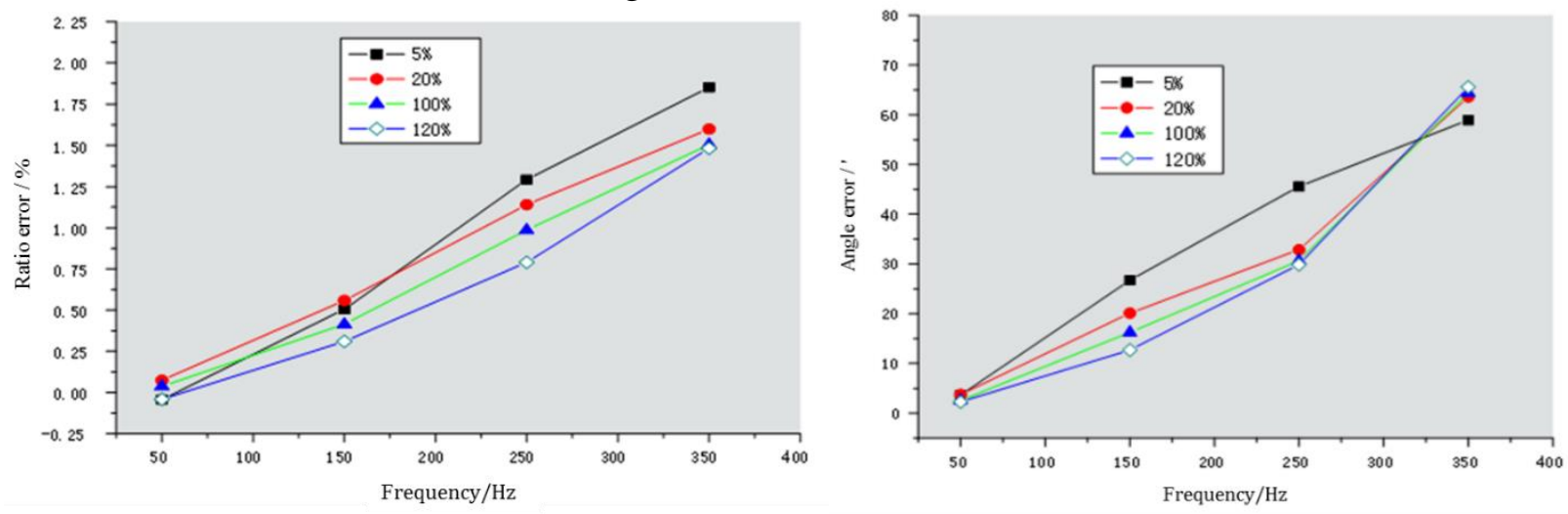

Fig. 10 The ratio error and angle error of active electronic current transformer caused by the harmonic

Under the condition of superposing the harmonic waves on the fundamental wave, the ratio error and angle error of the $150 \mathrm{~Hz}$ signal respectively are $0.3 \%$ and 12 '; the ratio error and angle error of the $250 \mathrm{~Hz}$ signal respectively are $0.7 \%$ and 29'; the ratio error and angle error of the $350 \mathrm{~Hz}$ signal respectively are $1.4 \%$ and 65 . The ratio error and angle error of electronic current transformer based on rogowski coil become larger with the increase of frequency.

\section{Conclusion}

Based on the analyzing the influence of temperature, primary wiring and harmonic on the measurement error of electronic current transformer, this paper carried out the corresponding experimental verification and obtained partial error characteristics of electronic voltage transformer. Conclusions are as follows:(1)The measurement error of all fiber optic current transformer is sensitive to the change of temperature and there is no obvious regularity. The fluctuation ranges of ratio error and angle error respectively are from $0.8 \%$ to $1.3 \%$ and from $30^{\prime}$ to $70^{\prime}$. But the measurement error of electronic current transformer based on rogowski coil is scarcely affected by temperature. The variations of ratio error and angle error are respectively $0.16 \%$ and 4 ' in the full temperature range. (2)The measurement error of fiber current transformer is affected greatly by the primary wiring. The influence of primary wiring on the error of active electronic current transformer can be neglected when the current is larger than $20 \%$ of the rated current. (3)With the increase of frequency, the variation of ratio error range become smaller gradually. But the angle error is on the contrary. The error of active electronic current transformer becomes larger with the increase of the frequency.

\section{Acknowledgement}

This research was financially supported by the State Grid Corporation of China Science and Technology Project (JL71-15-042).

\section{Reference}

[1] Bohnert K, Gabus P, Nehring J, et.al. Temperature and vibration insensitive fiber-optic 
current sensor [J].Journal of Lightwave Technology, 2002, 20(2): 267-276.

[2] WANG Peng, LUO Chengmu, ZHANG Guixin. Electronic current transformer based on lowpower current transformer [J]. Automation of Electric Power Systems, 2006, 30(4): 98-101.

[3] Fiber Optic Sensors Working Group. Optical current transducers for power systems: a review[J]. IEEE Trans. on Power Delivery, 1994, 9(4): 1778-1788.

[4] SANDERS G A, BLAKE J N, ROSE A H, et.al. Commercialization of fiber-optic current and voltage sensors at nxtphase[C]. The 15th Optical Fiber Sensors Conference Technical Digest. Portland, UK: OR, United States OFS, 2002: 31-34.

[5] SHEN Zhu, LUO Chengmu. Development of electronic current transformer [J]. Automation of Electric Power Systems, 2001, 25(22): 59-63.

[6] LUO Sunan, TIAN zhaobo, ZHAO xicai. Performance analysis of air-core current transformer [J]. Proceedings of the CSEE, 2004, 24(3): 108-113.

[7] ZHOU Wenzhong, ZHAO Guosheng, LI Haiyang. Error Analy-sis and Improved Method of Rogowski Coil [J]. Power System Protec-tion and Control, 2009, 37(20):99-103.

[8] LIU Bin, YE Guoxiong, GUO Keqin, et.al. Calculation Method of Composite Error for Electronic Current Transformers Based on Rogowski Coil [J]. High Voltage Engineering, 2011, 37(10): 2391-2397. 